\title{
A Study of Renal Resistive Index in Hepatic Cirrhosis
}

\author{
Vinodh $\mathbf{V}^{1}$, Shashidhar $\mathrm{G}^{2}$, M.S. Krishnamurthy ${ }^{3}$ \\ ${ }^{1}$ Senior Resident, ${ }^{2}$ Associate Professor, ${ }^{3}$ Professor and HOD, MVJ Medical College and Research Hospital, India
}

Corresponding author: Dr. Shashidhar G, Associate Professor, MVJ Medical College and Research Hospital, Dandupalya, Hoskote, Bangalore Rural 562114, India

DOI: $10.21276 / \mathrm{ijcmsr} .2018 .3 .3 .26$

How to cite this article: Vinodh V, Shashidhar G, M.S. Krishnamurthy. A study of renal resistive index in hepatic cirrhosis. International Journal of Contemporary Medicine Surgery and Radiology. 2018;3(3):C116-C120.

\section{A B S T R A C T}

Introduction: Hepatorenal syndrome is a form of functional renal failure without renal pathology that occurs in about $10 \%$ of patients with advanced cirrhosis or acute liver failure. In patients with cirrhosis the early renal impairment or renovascular vasoconstriction can be predicted by renal arterial resistance index (RI). The study was conducted to evaluate the intrarenal arterial changes in patients with hepatic cirrhosis to anticipate development of hepatorenal syndrome.

Material and Methods: A prospective study was conducted between October 2015 and August 2017 in a group of 59 and 30 cirrhotics as well as control group respectively.Special proforma was designed to enter all the basic informations about the patient, history of recent event and the past history, clinical examination and the relevant investigations such as liver function test, renal function test, complete blood count, urine examination, viral markers, ultrasonography of abdomen and the intrarenal artery Doppler for the resistive index (RI) calculation.

$\mathrm{RI}$ was calculated using the formula.

$\mathrm{RI}=$ (peak systolic flow- peak diastolic flow)/peak systolic flow

Results: The study result showed RI was significantly higher in patients when compared to healthy controls (0.63 vs 0.54 , $P<0.01)$. As compared to the controlled group without ascites RI was significantly greater in patients with ascites (0.63 vs $0.73, P<0.01)$. RI greater than 0.73 was significant independent predictor of subsequent HRS development $(P=0.006)$.

Conclusion: The study was concluded explaining the significant role of intrarenal RI measurement as a predictor of HRS and can be further validated for regular monitoring of cirrhotic patients at risk of developing renal impairment.

Key Words: Hepatic Cirrhosis, Renal Resistive Index, Hepatorenal Syndrome, Duplex Doppler Ultrasonography

\section{INTRODUCTION}

Renal failure is an outrageous complication of cirrhosis ${ }^{1,2,3}$ and liver transplantation is considered as one of the important prognostic factor in such cases. Patients with cirrhosis and renal failure are more prone to die, while awaiting transplantation and have increased morbidity and mortality post transplantation, as compared with those without renal failure. . $^{1,4}$

Hepatorenal syndrome (HRS) is a functional renal failure in cirrhotic patients with pathophysiology similar to prerenal azotemia. The annual frequency of HRS in cirrhotic patients with ascites has been described from $8 \%$ to as high as $40 \% .{ }^{6,7}$ There is considerable evidence that interactions between systemic and portal hemodynamics lead to intense renal vasoconstriction and HRS. ${ }^{1,2,8}$

The notion that serum creatinine measurements accurately indicate renal dysfunction in cirrhosis is a fallacy. Renal arterial vasoconstriction may persist for weeks, even months before an increase in blood urea nitrogen or serum creatinine is evident ${ }^{9}$ Therefore it is very prudent to identify renal failure at an early stage by sensitive methods so that appropriate measures can be taken. And renal RI (RRI) is an early indicator of HRS even before creatinine could rise to fulfil the criteria for HRS. ${ }^{10}$
Duplex Doppler ultrasonography of the kidneys is a noninvasive tool to assess blood flow and arterial vascular resistance as a parameter for vasoconstriction ${ }^{11,12}$

The arterial resistance index (RI) serves to detect early renal function impairment in Cirrhotic patients. The role of vasoconstriction in the pathogenesis of cirrhotic kidney disease has been confirmed by correlation between increased RI and azotemia. ${ }^{13}$ A positive correlation has also been described between RI and plasma renin activity as well as plasma aldosterone concentration. ${ }^{14}$

Color and power Doppler can provide an accurate morphological and functional evaluation of the intraparenchymal vascularity and detect reduced or no blood flow in the kidney or in a portion of the kidney. In this case, there will bicolor signals from the undamaged part of the kidney but not from the ischemic part. The use of contrast agent increases diagnostic confidence in this type of lesions. ${ }^{15}$ Incidence of Hepatorenal syndrome develops in 5\% of patients with chronic liver disease who present with upper gastrointestinal bleeding, 30\% of patients admitted with spontaneous bacterial peritonitis, $10 \%$ of patients with ascites treated with total paracentesis, and $25 \%$ of patients with severe alcoholic hepatitis. The probability of hepatorenal syndrome developing in a patient with cirrhosis 
and new onset of ascites is $7-10 \%$. The 5 -year probability of hepatorenal syndrome developing in a patient with cirrhosis and recurrent ascites is $40 \%$. $^{16,17,18}$

Study aimed to assess the value of RRI in Hepatic cirrhosis. To study association between RRI and Hepatic cirrhosis and to study association between RRI and Hepatorenal syndrome.

\section{MATERIAL AND METHODS}

A prospective study has been conducted in a time period between October 2015 and august 2017 in MVJ Medical College and research hospital on 56 cirrhotics with an age limit of 30-70 years those who are clinically and sonologically diagnosed with cirrhosis. Patients with other comorbidities such as hypertension, diabetes mellitus, previous renovascular disease, vascular disease that affect kidneys, and congenital or acquired renal abnormalities were excluded. As well as patients those who underwent kidney transplantation, and exposed to nephrotoxic drug, alcohol and also those who had decreased kidney size were also excluded from the study.

A proforma was prepared which included detailed history,clinical examination and requisite investigations. Where detailed history regarding the comorbidities, social history clinical signs and symptoms, and laboratory investigations that are relevant for the study.

Duplex Doppler evaluation of the renal arteries was done using a $3.5 \mathrm{MHz}$ convex transducer (GE Voluson 730 pro). The patients were asked to fast at least $4 \mathrm{~h}$ before examination to reduce masking by intestinal gas. Doppler signals were taken from interlobar arteries and arcuate arteries in both kidneys. Colour Doppler ultrasound was used to help to identify the arteries. A train of atleast three similar, sequential time-velocity waveforms of Doppler

signals was obtained at each point of measurement during suspended respiration.

The RI was calculated with the formula RI = (peak systolic velocity - end diastolic velocity)/ peak systolic velocity. Patients were excluded if it was not possible to measure the $\mathrm{RI}$ in two different places in each kidney due to massive ascites or masking by gas. Inter-observer variability was kept to the minimum by having the same ultrasonologist perform the Doppler studies.

eGFR using:

MDRD:

eGFR $(\mathrm{mL} / \mathrm{min}$ per $1.73 \mathrm{~m} 2)=186 \mathrm{x} \mathrm{Se} \mathrm{Cr}(-1.154) \mathrm{x}$ age $(-0.203) \times(0.742$ if female $)$

Cockcroft-Gault:

$\mathrm{CrCl}(\mathrm{mL} / \mathrm{min})=$

(140 - age (years) x weight $(\mathrm{kg}) \times$ [0.85 if female $]) /(72 \times \mathrm{Se}$ $\mathrm{Cr}(\mathrm{mg} / \mathrm{dL})$

The master chart provided as shows the data obtained in both Cases and Controls.

\section{STATISTICAL ANALYSIS}

Descriptive and inferential statistical analysis has been carried out in the present study. Results on continuous measurements are presented on Mean SD (Min-Max) and results on categorical measurements are presented in Number (\%). Significance is assessed at 5\% level of significance.
The following assumptions on data were made, Assumptions:

1. Dependent variables should be normally distributed

2. Samples drawn from the population should be random, Cases of the samples should be independent

Analysis of variance(ANOVA) has been used to find the significance of study parameters between three or more groups of patients, Student t test (two tailed, independent) was used to find the significance of study. Chi-square/ Fisher Exact test has was to find the significance of study parameters on categorical scale between two or more groups.

Significant figures

+ Suggestive significance ( $\mathrm{P}$ value: $0.05<P<0.10)$

* Moderately significant ( $\mathrm{P}$ value: $0.01<P<0.05$ )

** Strongly significant (P value: $P$ 0.01)

Statistical software: The Statistical software namely SAS 9.2, SPSS 15.0, Stata 10.1,

MedCalc 9.0.1, Systat 12.0 and R environment ver.2.11.1 were used for the analysis of the data and Microsoft word and Excel have been used to generate graphs, tables etc.

\section{RESULT}

In the present study, 56 cirrhotic cases (14 without ascites and 42 with ascites) and

30 Non cirrhotic controls were studied. A total of 86 patients who were split into three groups that consisted of 14 patients who had cirrhosis without ascites, 42 patients who had cirrhosis with ascites and 30 patients who are with noncirrhosis controls. Based on the results, $75 \%$ of the patients had cirrhosis with ascites when compared to $25 \%$ of the patients who had cirrhosis without ascites. Within the overall population within Groups 1 and 2, male patients dominated the gender wise distribution study. Further analysis reveal that a significant amount of the males (85.71\%) had cirrhosis with ascites out of the total number of patients who had cirrhosis with ascites. Noticeably, the number of female patients ranked second overall within the two groups with only 2 female patients having Cirrhosis without ascites. Control groups had 50/50 patient distribution for both male and female patients. The Groups were further broken down by Age distribution ranging from ages 31 through 70 . The age distribution with the highest number of patients was between ages 41 to 50; where approximately 14 patients had cirrhosis with ascites and the number stayed steady at 14 patients for control group as well. Coincidently, the age distribution with the lowest patient count was ages 31 through 40 and 61 through 70 with only 2 patients having Cirrhosis without ascites, as well as 2 patients ages 61 through 70 within the Control group. Further study was conducted on the distribution of Etiology within the groups, where the highest concentration of patients were located in Group 2 with 26 patients with Cirrhosis with ascites using Alcohol and on the other side with Group 1 - Cirrhosis without ascites having 1 patient using $\mathrm{HCV}$ and 1 patient using Cryptogenic. 85.71\% of the patients having Cirrhosis with ascites exhibited pain in the abdomen. 29 patients out of 42 patients who had showed signs of jaundice. 13 patients within Group 2 also showed some sign of brain diseases which was still higher when compared to the total population within Group 1 and 


\begin{tabular}{|l|c|c|c|}
\hline Resistive Index & Group 1 & Group 2 & controls \\
\hline & $\begin{array}{c}\text { Cirrhosis } \\
\text { without } \\
\text { ascites }\end{array}$ & $\begin{array}{c}\text { Cirrhosis } \\
\text { with ascites }\end{array}$ & \\
\hline Sundeep et al $^{1}$ & $0.62 \pm 0.06$ & $0.74 \pm 0.04$ & $0.52 \pm 0.03$ \\
\hline Yaseer et al $^{19}$ & $0.63 \pm 0.03$ & $0.70 \pm 0.03$ & \\
\hline Present study & $0.63 \pm 0.03$ & $0.73 \pm 0.03$ & $0.54 \pm 0.02$ \\
\hline Table-1: Comparison of RRI in Cirrhosis with Other Studies & \\
\hline
\end{tabular}

\begin{tabular}{|l|c|c|}
\hline Median serum creatinine & $\begin{array}{c}\text { Cirrhosis with- } \\
\text { out ascites } \\
\mathbf{n = 1 4}\end{array}$ & $\begin{array}{c}\text { Cirrhosis with } \\
\text { ascites } \\
\mathbf{n = 4 2}\end{array}$ \\
\hline Sundeep et al & 1 & 1.4 \\
\hline Yasser et al & 1.1 & 1.4 \\
\hline Present Study & $1.04 \pm 0.20$ & $1.55 \pm 0.81$ \\
\hline \multicolumn{2}{|c|}{ Table-2: Comparison of median serum creatinine in cirrhosis } \\
with other studies
\end{tabular}

2, Group 2 also had the highest number of patients showing signs of gastrointestinal bleeding. To conclude, Group 2 had the highest number of patients exhibiting a wide array of problems ranging from child pugh score to eGFR MDRD and Serum Sodium and Serum Creatinine and, Serum Albumin counts when compared to other groups used for this study. Due to these varies problems Group 2 faced, this indirectly also increased the 3 month Morality Percentage when compared to other Groups.

\section{DISCUSSION}

This study was done to evaluate the role of renal resistive index in cirrhosis with special reference to ascites. In view of the factors which influence RRI in cirrhosis correlation with serum creatinine, serum albumin, total bilirubin, INR, eGFR, MELD score, and CPT score was analysed (Table 1). 56 cirrhotic patients were studied. Hypertensives, Diabetics and patients with history of renal toxic drugs were excluded from the study, as these medications are known to alter the intrarenal hemodynamics by blocking the Renin-angiotensin system activation and reduce the renal vascular resistance, thus altering the resistive index values. In our study most common cause of cirrhosis was alcohol, followed by hepatits $\mathrm{B}$ infection.Sundeep et $\mathrm{a}^{1}{ }^{1}$ study also showed $50 \%$ of patients having alcohol as etiological factor. ${ }^{1}$ Clinical presentation, clinical signs and complications. The commonest clinical feature in our study was pain abdomen (79\%), followed by abdominal distension (63\%) and jaundice (70\%).The other clinical features in our study included fever, nausea, vomiting,breathlessness and swelling of lower limbs. In study by Nand et al in which Abdominal distension (78\%) was commonest feature followed by jaundice (60\%) and Abdominal pain (55\%). ${ }^{20}$ In a study by Sen AK et al most common clinical presentation was abdominal distension followed by swelling of feet (51.45\%) and Jaundice was seen in $49.28 \%$ cases. ${ }^{21}$

In our study $69 \%$ of group 2 patients with mean RI 0.73 had Jaundice as compared to group 1 of mean RI 0.63 of where $29 \%$ patients had jaundice.In our study history of Gastrointestinal bleeding was most frequently present in group 2 where cirrhotic patients had ascites. In a study done by zaman et al shows that 9 to $35 \%$ of ciirhosis patients have history of GI bleed. ${ }^{22}$ In our study Hepatic encephalopathy occurs most commonly in in group 2 where patients had cirrhosis with ascites with mean RI $=0.72$. Similar results were observed by sundeep et al. ${ }^{1}$ On the basis of previous studies intrarenal RI of 0.70 was considered as a threshold value being indicative of increased renal vasoconstriction. In our study we noted that intrarenal RI values were significantly increased in non-ascitic cirrhotic patients in comparison to healthy controls and even higher values were noted inascites patients in absence of elevated serum creatinine.RI progressively increased as the patients moved from cirrhosis without ascites, to ascites and then to HRS. Elevated RI values were noted in $16 \%$ cirrhosis patients without ascites and $60 \%$ patients with decompensated cirrhosis. In the literature, other studies also showed higher RI levels in cirrhotic patients with ascites than in non-ascitic subjects. In patients with cirrhosis, RI was significantly greater in patients with ascites

than those without ascites ( 0.73 vs. $0.63, \mathrm{p}<0.01)$. $\mathrm{RI}>0.73$ was significant independent predictor of subsequent HRS development $(\mathrm{p}=0.006)$ We followed few patients for 6 months after the enrolment and found that later renal outcome was significantly worse in those with RI elevated $>0.70$. The mean baseline RI was significantly high in patients who developed HRS subsequently than patients who did not $(0.73$ vs. 0.63$)(\mathrm{p}<0.05)$.

Gotzberger et al investigated RI levels in liver cirrhosis patients with or without ascites, fatty liver disease and healthy control subjects. ${ }^{12,23}$ RI was significantly higher in ascitic patients compared to nonascitic patients ( 0.74 vs. $0.67, \mathrm{p}<0.01)$ and in non-ascitic patients with livercirrhosis than in control subjects (0.67 vs. $0.62, \mathrm{p}<0.01)$. Also $48 \%(19 / 40)$ of patients with liver cirrhosis and normal serum creatinine concentration showed elevated RI levels. Therewere no significant differences in RI levels between patients with fatty liver disease and controls (0.63 vs. 0.62). This findings were consistent with findings of study done by sundeep et al and yaseer et al RI progressively increased as the patients moved from cirrhosis without ascites, to ascites and then to HRS. ${ }^{1,19}$ In our study mean total bilirubin was elevated much more in patients with ascites as compared to non ascitic cirrhosis patients. Study done by Gotzberger et al and Yasser et al also showed similar results. ${ }^{12,19}$ The difference in serum albumin levels was not significant $(\mathrm{p}=0.275)$. In our study serum albumin was decreased in both groups without a significant difference. Median serum creatinine values were higher in group $2[1.55 \mathrm{mg} / \mathrm{dl}$ vs. $1.04 \mathrm{mg} / \mathrm{dl}$ ( $p>0.087)$. In our study serum creatinine was raised in patients with ascites (Table 2) as compared with patients without ascites who had marginally elevated serum creatinine or can have normal levels also. eGFR MDRD was significantly different $(\mathrm{P}=$ 0.013 ) between cirrhotic patients with and without ascites our study shows that cirrhotic patients with ascites had low eGFR compared to without ascites patients. 100\% patients with $\mathrm{RI}=0.73$ belonged to $\mathrm{B}$ or $\mathrm{C}$ class but none of patients in class A where as in patients with RI $<0.72$ only $14 \%$ of patients belonged to A class but none of the patient in class 
C.Our study shows that when RI $>73$, most of patients have high child pugh score with increase 3 month mortality percentage.As compared with study done by sundeep et al, ${ }^{1}$ also showed that $64 \%$ of patients in class $\mathrm{A}$ had $\mathrm{RI}<0.72$ and $56 \%$ of patients in class $\mathrm{C}$ had RI $=0.72$ Model for end-stage liver disease (MELD) score MELD score was significantly different $(P=0.005)$ between cirrhotic patients with and without ascites.

\section{Limitations of the study}

Since there is no definitive cut-off for RI in HRS, repeat renal Doppler was not done in all patients with raised RI but normal creatinine. This may have been indicated in patients who were progressing into HRS.

\section{CONCLUSION}

Renal failure is a common problem among patients with liver cirrhosis and is found to be occurring due to intrarenal vasoconstriction. The most sustainable strategy of treatment to quantify intra-renal vascular resistance would be the use of Doppler ultrasound measurement of the RI in cirrhotic patients before HRS develops.Circulatory dysfunction, arterial underfilling and increased endogenous vasoconstrictor activity that affect the intrarenal circulation makes patients with cirrhosis particularly prone to renal failure.An important impractical method for routine clinical purposes would be the measurement of glomerular filtration rate (GFR) on the basis of the clearance of inulin or radioisotopic substances which is considered to be more accurate. The current definition of renal failure in cirrhosis identifies only those patients with a severely reduced GFR ( $<30 \mathrm{ml}$ per minute) due to Serum creatinine elevation which is a delayed piece of HRD spectrum.Doppler ultrasound measurement of the $\mathrm{RI}$ is also useful to quantify renovascular resistance in cirrhotic patients.In our study we noted that intrarenal RI values were significantly increased in nonascitic cirrhotic patients in comparison to healthy controls and even higher values were noted in ascites patients in absence of elevated serum creatinine.RI progressively increased as the patients moved from cirrhosis without ascites, to ascites and then to HRS. This study shows that renal Doppler RI is a useful non-invasive tool for indicating the presence of hepatorenal syndrome as well as acting as a prognostic indicator in patients with cirrhosis of the liver.

\section{REFERENCES}

1. Sundeep Goyal, Dixit VK, Jain AK,Shukla RC, Jayant Ghosh,Vinod Kumar. Intrarenal resistance index as a predictor of early renal impairment in patients with liver cirrhosis. Tropical gastroenterology 2013; 34(4)

2. Gines P, Guevara M, Arroyo V, Rodes J. Hepatorenal syndrome. Lancet. 2003;362(2):1819-27.

3. Moreau R, Lebrec D. Acute renal failure in patients with cirrhosis: perspectives in the age of MELD. Hepatology. 238 Tropical Gastroenterology 2013;34(4):235-239 2003;37:233-43.

4. Nair S, Verma S, Thuluvath PJ. Pretransplant renal function predicts survival in patients undergoing orthotopic liver transplantation. Hepatology. 2002;35 (4):1179-85.
5. Gonwa TA, Klintmalm GB, Levy M, Jennings LS, Goldstein RM, Husberg BS. Impact ofpretransplant renal function on survival after liver transplantation. Transplantation. 1995;59 (6):361-5.

6. Arroyo V, Fernandez J, Gines P. Pathogenesis and treatment of hepatorenal syndrome. Semin Liver Dis. 2008;28 (1):81-95.

7. Munoz SJ. The hepatorenal syndrome. Med Clin North Am. 2008;92 (3):813-37.

8. Arroyo V, Colmenero J. Ascites and hepatorenal syndrome in cirrhosis: pathophysiological basis of therapy and current management. J Hepatol. 2003;38Suppl 1:S69-89. 74

9. Platt JF, Ellis JH, Rubin JM, Merion RM, Lucey MR. Renal duplex Doppler ultrasonography: a noninvasive predictor of kidney dysfunction and hepatorenal failure in liver disease. Hepatology. 1994;20 (5):362-9.

10. Aslam M, Ram SA, Krishnamurthy A. The renal resistive index is a non invasive indicator of hepatorenal syndrome in cirrhotics. J AdvClin Res Insights 2016;3 (6):23-27.

11. Berzigotti A, Casadei A, Magalotti D, Castaldini N, Losinno F, Rossi C, et al. Renovascular impedance correlates with portal pressure in patients with liver cirrhosis. Radiology. 2006;240 (4):581-6.

12. Gotzberger M, Kaiser C, Landauer N, Dieterle C, Heldwein W, Schiemann U. Intrarenal resistance index for the assessment of early renal function impairment in patients with liver cirrhosis. Eur J Med Res. 2008;13 (5):383-7.

13. Sacerdoti D, Bolognesi M, Merkel C, Angeli P, Gatta A. Renal vasoconstriction in cirrhosis evaluated by duplex Doppler ultrasonography. Hepatology. 1993;17 (2):219-24.

14. Kastelan S, Ljubicic N, Kastelan Z, Ostojic R, Uravic $\mathrm{M}$. The role of duplex doppler ultrasonography in the diagnosis of renal dysfunction and hepatorenal syndrome in patients with liver cirrhosis. Hepatogastroenterology. 2004;51 (3):1408-12.

15. Wilensky A. Occurrence, Distribution and Pathogenesis of So-Called Liver Death and/or the Hepatorenal Syndrome. Arch Surg 1939;38 (5): 625-691

16. D. Popov, R. Krasteva, R. Ivanova, L. Mateva,Z. Krastev. Doppler Parameters of Hepatic and Renal Hemodynamics in Patients with Liver Cirrhosis 1. International Journal of Nephrology 2012; 2012 (Article ID 961654)

17. Anthony P, Ishak K.G, Nayak N.C, Poulsen H.E, Schever P.J, Subin L.H Bullitin of World Health Organisation The morphology of liver: definition, nomenculature and classification 1977 p 521-540

18. V. Arroyo, P. Ginès, A. L. Gerbes et al. Definition and diagnostic criteria of refractory ascites and hepatorenal syndrome in cirrhosis. Hepatology, 1996;23(3):164-176.

19. Yasser M Fouad, Hamdy Mokarrab, Ahmed Fathi Elgebaly. Renal duplex doppler ultrasound in patients with HCV related liver cirrhosis. Tropical Gastroenterology 2009; 30(4) 90.

20. Nand N, Malhotra P, Dhoot DK. Clinically profile of alcoholic liver disease in a tertiary care centre and its correlation with type, amount and duration of alcohol 
consumption. J Assoc physicians india 2015; 63(3):142091.

21. Sen AK, Doley RM, Jerang O, et al. clinical profile of patients with alcoholic liver disease in upper assam of north east india. J. Evid. Based Med. Healthc. 2017; 4 (40):2427-2431.

22. Zaman A, Hapke R, Flora K, Rosen HR, Benner K. Factors predicting the presence of esophageal or gastric varices in patients with advanced liver disease. Am J Gastroenterol 1999; 94(5): 3292-3296

23. Götzberger M, Singer J, Kaiser C, Gülberg V. Intrarenal resistance index as a prognostic parameter in patients with liver cirrhosis compared with other hepatic scoring systems. Digestion 2012;86(1):349-54.

Source of Support: Nil; Conflict of Interest: None

Submitted: 16-07-2018; Accepted: 18-08-2018; Published online: 01-09-2018 